$$
\text { Pontifícia } \text { Universidade }_{\text {do Rio de Janeiro }}
$$

\title{
UM MODELO INTEGRADOR PARA FORMULAÇÃO DE ESTRATÉGIAS MÚLTIPLAS: Contribuição da Análise Prospectiva
}

Tese de Doutorado

Tese de Doutorado apresentada ao Programa de Pós-graduação em Engenharia de Produção do Departamento de Engenharia Industrial da PUC-Rio como requisito parcial para obtenção do grau de Doutor em Engenharia de Produção.

Orientadora: Maria Angela Campelo de Melo

Co-orientador: Nélio Domingues Pizzolato

Rio de Janeiro

Fevereiro de 2011 


$$
\text { Pontifícia } \underbrace{}_{\text {Niversidade }} \text { C }_{\text {datólica }}
$$

Andréa Belfort de Andrade Santos

\section{Um Modelo Integrador para Formulação de Estratégias Múltiplas: Contribuição da Análise Prospectiva}

Tese apresentada como requisito parcial para obtenção do grau de Doutor pelo Programa de Pós-Graduação em Engenharia de Produção da PUC-Rio. Aprovada pela Comissão Examinadora abaixo assinada.

Prof. Maria Angela Campelo de Melo

Orientadora

Departamento de Administração - PUC-Rio

Prof. Nélio Domingues Pizzolato

Co-orientador e Presidente

Departamento de Engenharia Industrial - PUC-Rio

Prof. Teresia Diana Lewe van Aduard de Macedo-Soares

Departamento de Administração - PUC-Rio

Prof. Eduardo Marques

FGV-RJ

Prof. Paulo Roberto de Mendonça Motta

FGV-RJ

Prof. Walter Fernando Araújo de Moraes

Departamento de Ciências Administrativas - UFPE

Prof. José Eugenio Leal

Coordenador Setorial do Centro Técnico Científico - PUC-Rio

Rio de Janeiro, 2 de fevereiro de 2011 
Todos os direitos reservados. É proibida a reprodução total ou parcial do trabalho sem autorização da universidade, do autor e da orientadora.

\section{Andréa Belfort de Andrade Santos}

Engenheira Civil pela Universidade Federal Fluminense, M.Sc. em Engenharia de Produção pela PUC-Rio (1996) e cursos de especialização em Administração Financeira pela FGV-RJ (1998) e em Economia da Energia pela COPPEAD/Instituto de Economia-UFRJ (2003). Especialista em estratégia empresarial e construção de cenários, com 15 anos de experiência em consultoria. Consultora sênior da Macroplan ${ }^{\circledR}-$ Prospectiva, Estratégia e Gestão. Participou como consultora técnica/ gerente de mais de 50 projetos de consultoria em estratégia empresarial; construção de cenários macroeconômicos e setoriais; gestão orientada para resultados; e elaboração, implantação e monitoramento de planos estratégicos e de negócios em diversos setores, notadamente no setor energético.

Ficha Catalográfica

Santos, Andréa Belfort de Andrade

Um modelo integrador para formulação de estratégias múltiplas: contribuição da análise prospectiva / Andréa Belfort de Andrade Santos ; orientadora: Maria Angela Campelo de Melo ; co-orientador: Nélio Domingues Pizzolato. - 2011.

264 f. : il. (color.) ; $30 \mathrm{~cm}$

Tese (doutorado) - Pontifícia Universidade Católica do Rio de Janeiro, Departamento de Engenharia Industrial, 2011.

Inclui bibliografia

1. Engenharia Industrial - Teses. 2. Análise prospectiva. 3 . Planejamento estratégico. 4. Planejamento sob cenários. 5. Aprendizagem organizacional. 6. Planejamento adaptativo. 7. Pesquisa-ação. 8. Eletrobras. I. Melo, Maria Angela Campelo de. II. Pizzolato, Nélio Domingues. III. Pontifícia Universidade Católica do Rio de Janeiro. Departamento de Engenharia Industrial. IV. Título. 
À minha família, meu marido Marcio, meu filho Rafael, meus pais Ernani Gabriel e Maria Luiza e meus irmãos Flávia, Rogério, Luciano e Rosana. 


\section{Agradecimentos}

à Professora Maria Angela Campelo de Melo, pela grande amizade e confiança, pelo apoio e estímulo, e por guiar meus passos no desenvolvimento deste trabalho;

ao Professor Nélio Pizzolato, pela co-orientação e pelo apoio;

aos Professores Eduardo Marques e Teresia Diana L.V.A. de Macedo-Soares, pela especial colaboração no exame de qualificação e na defesa do projeto desta Tese;

aos professores e funcionários do Departamento de Engenharia Industrial da PUCRio, em especial à Cláudia Guimarães Teti e Fernanda Frias, pela vigilância constante dos prazos e datas críticas;

aos consultores Claudio Porto e Aluisio Guimarães, pela amizade, pelo incentivo constante e pelas discussões profícuas no decorrer do trabalho;

aos colegas da Macroplan, pelo estímulo e pela troca de conhecimentos e experiências ao longo dos últimos quinze anos;

à Eletrobras, por permitir a realização do estudo de caso e pela disponibilidade para experimentar inovações metodológicas no seu processo de planejamento;

à CAPES, pelo apoio financeiro nos dois primeiros anos do curso;

à Coordenação Central de Pós-Graduação da PUC pela bolsa concedida; e em especial, aos meus pais, Ernani e Maria Luiza, cujas vidas inspiraram a minha, ao meu marido Marcio, ao meu filho Rafael e aos meus irmãos, Flávia, Rogério, Luciano e Rosana, pela compreensão, apoio, carinho e torcida sempre presentes. 


\section{Resumo}

Santos, Andréa Belfort de Andrade; Melo, Maria Angela Campelo (Orientadora). Um Modelo Integrador para Formulação de Estratégias Múltiplas: Contribuição da Análise Prospectiva. Rio de Janeiro, 2011. 264p. Tese de Doutorado - Departamento de Engenharia Industrial, Pontifícia Universidade Católica do Rio de Janeiro.

Para lidar com os graus cada vez mais elevados de dinamismo e complexidade do ambiente empresarial, com altos níveis inerentes de incerteza, as organizações têm desenvolvido processos de planejamento baseados na análise prospectiva, trazendo para a discussão estratégica um pensamento plural sobre o futuro. No entanto, na hora de formular as estratégias e definir os comprometimentos da empresa, essa diversidade, explorada na análise de cenários, é negligenciada e o processo de planejamento desenrola-se como se o mundo caminhasse em uma direção única. Para isso, contribui o distanciamento entre a construção de cenários e a formulação de estratégias nos processos de planejamento sob cenários. Visando potencializar a interligação entre essas áreas, objetiva-se, neste trabalho, avaliar o processo de formulação de estratégias e as possibilidades de contribuição da análise prospectiva a esse processo. Com esse fim, propõe-se um Modelo Integrador da Análise Prospectiva e da Estratégia, desenvolvido seguindo a metodologia de pesquisa-ação, o qual foi formulado com sólida fundamentação teórica nas áreas de estratégia, análise prospectiva e aprendizagem organizacional e aplicado durante o processo de planejamento corporativo do Sistema Eletrobras. O Modelo busca consolidar um processo de formulação de estratégias múltiplas enriquecido com o aporte dos cenários, constituindo um instrumento relevante para a gestão da incerteza na tomada de decisão da organização.

\section{Palavras-chave}

Análise prospectiva; planejamento estratégico; planejamento sob cenários; aprendizagem organizacional; planejamento adaptativo; pesquisa-ação; Eletrobras. 


\section{Abstract}

Santos, Andréa Belfort de Andrade; Melo, Maria Angela Campelo (Advisor). An Integrative Model for Multiple Strategies Formulation: the Contribuition of Strategic Foresight. Rio de Janeiro, 2011. 264p. Doctoral Dissertation - Departamento de Engenharia Industrial, Pontifícia Universidade Católica do Rio de Janeiro.

In order to deal with the corporate environment's high degree of dynamism, complexity and uncertainty, organizations develop planning processes based on prospective approach, incorporating into the strategic discussion a plural reflection on the future. However, in corporate practice it is not usual to make extensive use of complex studies of scenarios when formulating the firm's strategies and defining its commitments. This is due to the decision-making difficulties encountered by managers when confronted with these processes' diverse possibilities and the distance that exists between scenario construction and strategy formulation. In order to enhance the interaction between strategy formulation and prospective approach, this thesis proposes an Integrative Model for multiple strategies formulation, grounded on solid theoretical foundations in the areas of strategic foresight, strategy and organizational learning, which was developed following the methodology of action- research and applied on the corporate planning process of Sistema Eletrobras, a business group in Brazil's electricity sector. The Model seeks to consolidate a multiple strategy formulation process, enriched by support from scenarios, and constitutes an important instrument for the management of uncertainty in decision-making.

\section{Keywords}

Strategic foresight; strategic planning; scenario planning; adaptive planning; organizational learning; action-research; Eletrobras. 


\section{Sumário}

$\begin{array}{lc}\text { Resumo } & 6\end{array}$

$\begin{array}{ll}\text { Abstract } & 7\end{array}$

$\begin{array}{lr}\text { Lista de Figuras } & 12\end{array}$

$\begin{array}{ll}\text { Lista de Quadros } & 14\end{array}$

$\begin{array}{ll}\text { Lista de Tabelas } & 15\end{array}$

$\begin{array}{ll}\text { 1. Introdução } & 16\end{array}$

$\begin{array}{ll}\text { 1.1. } & \text { Caracterização do Problema de Pesquisa } \\ & 17\end{array}$

$\begin{array}{ll}\text { 1.1.1. Objetivos da Pesquisa } & 18\end{array}$

$\begin{array}{ll}\text { 1.2. Referencial Teórico } & 19\end{array}$

$\begin{array}{ll}\text { 1.3. } & \text { Estrutura da Tese }\end{array}$

2. Investigando o Futuro 23

2.1. Caracterizando a Incerteza 24

$\begin{array}{ll}\text { 2.2. Previsão versus Prospectiva } & 31\end{array}$

$\begin{array}{ll}\text { 2.3. Prospectiva Estratégica } & 36\end{array}$

2.3.1. Estudos de Cenários: Conceitos e Tipologia 39

2.3.2. Contribuição dos Estudos de Cenários para a Prática
Estratégica

2.3.3. A Evolução dos Estudos de Cenários e Principais Metodologias 49

$\begin{array}{ll}\text { 2.3.3.1. A Escola Francesa } & 54\end{array}$

$\begin{array}{ll}\text { 2.3.3.2. A Escola Anglo-Saxônica } & 58\end{array}$

2.3.3.3. Semelhanças e Diferenças entre as Escolas 62

2.4. Compreendendo o Futuro 64 
3. A Estratégia em Condições de Incerteza 66

3.1. O que é Estratégia? 66

3.2. Processos de Formulação e Formação de Estratégia 77

$\begin{array}{lll}\text { 3.2.1. As Origens do Planejamento } & 78\end{array}$

3.2.2. As Escolas de Pensamento Estratégico 81

3.2.3. Ferramentas de Análise Estratégica 90

3.2.3.1. Análise SWOT - Strenghts, Weaknesses, Opportunities and 90 Threats

$\begin{array}{ll}\text { 3.2.3.3. Análise de Stakeholders } & 98\end{array}$

3.2.4. O Planejamento como Aprendizado 105

$\begin{array}{ll}\text { 3.2.4.1. Planejamento Adaptativo } & 105\end{array}$

$\begin{array}{ll}\text { 3.2.4.2. Aprendizagem Organizacional } & 111\end{array}$

3.3. Planejamento Estratégico Baseado em Cenários 123

3.3.1. O Método Desenvolvido por Godet e Giget 125

3.3.2. A Estrutura TAIDA ${ }^{\mathrm{TM}}$ do Planejamento Baseado em Cenários 127

$\begin{array}{ll}\text { 3.3.3. Da Incerteza à Estratégia } & 130\end{array}$

$\begin{array}{ll}\text { 3.3.4. Uso dos Cenários para a Escolha Estratégica } & 138\end{array}$

$\begin{array}{ll}\text { 3.4. A Estratégia diante da Incerteza } & 144\end{array}$

4. O Modelo Integrador - dos Cenários às Estratégias Múltiplas 147

4.1. Concepção do Modelo Integrador 149

$\begin{array}{ll}\text { 4.1.1. Detalhamento do Modelo } & 154\end{array}$

4.1.1.1. A Escolha Estratégica em Face dos Cenários 156

$\begin{array}{ll}\text { 4.1.1.2. Definição do Portfolio de Experimentos } & 160\end{array}$

$\begin{array}{ll}\text { 4.2. Implicações do Modelo para a Organização } & 161\end{array}$

4.3. Dos Cenários às Estratégias Múltiplas 166

5. Como o Trabalho foi Realizado: Aspectos Metodológicos 168 
5.1. A Pesquisa Bibliográfica: Arcabouço Teórico 169

5.2. A Pesquisa Aplicada: Estudo de Caso 169

$\begin{array}{ll}\text { 5.2.1. A Pesquisa-Ação } & 172\end{array}$

5.2.2. Críticas ao Método de Estudo de Caso e da Pesquisa-Ação 177

5.2.3. Delimitação do Estudo de Caso Baseado na Pesquisa-Ação 183

5.2.3.1. Planejamento da Pesquisa Experimental 186

5.2.3.2. Aplicação do Modelo Integrador e Avaliação após a Aplicação 190 de cada Módulo

$\begin{array}{lll}\text { 5.3. A Metodologia Empregada } & 191\end{array}$

6. O Modelo Integrador na Prática: o Planejamento Estratégico do Sistema Eletrobras $\quad 193$

6.1. O Sistema Eletrobras e o Planejamento Estratégico 193

$\begin{array}{lll}\text { 6.1.1. Antecedentes do Planejamento Estratégico } & 196\end{array}$

$\begin{array}{ll}\text { 6.2. Aplicação do Modelo Integrador: Formulação do Plano } & 199 \\ \text { Estratégico do Sistema Eletrobras 2010-2020 } & \end{array}$

6.2.1. Módulo I - Construção dos Cenários 199

6.2.2. Módulo II - Análise do Ambiente Interno e Ferramentas de 206
Avaliação Estratégica

6.2.2.1. Análise do Ambiente Interno 207

6.2.2.2. Análise da Estrutura da Indústria aplicada ao Negócio Geração 209

6.2.2.3. Análise de Stakeholders do Sistema Eletrobras 212

6.2.2.4. Análise SWOT para o Sistema Eletrobras 214

6.2.3. Módulo III - Formulação de Estratégias Múltiplas 217

6.2.4. Módulo IV - Análise de Consistência e de Robustez do Plano 221

6.3. Avaliação da Aplicação do Modelo 224

6.4. Revisitando o Modelo Integrador 226

$\begin{array}{lll}\text { 7. Conclusão } & 231\end{array}$

$\begin{array}{lll}\text { 8. Referências Bibliográficas } & 237\end{array}$ 
$\begin{array}{lll}\text { Anexo I. } & \text { Permissão da Eletrobras para a Pesquisa } & 249\end{array}$

Anexo II. Método de Análise Estrutural - Método MICMAC®

Anexo III. Checklist para Avaliar a Robustez e a Adaptabilidade das 252 Estratégias

Anexo V. Aplicação dos Questionários 255

1. Avaliação Após a Realização dos Módulos 255

2. Avaliação Posterior à Conclusão do Processo de
Planejamento Estratégico Corporativo

Anexo VI. Objetivos Estratégicos e Estratégias Específicas associadas 262 


\section{Lista de Figuras}

Figura 1: Arcabouço Teórico 20

Figura 2: Níveis de Turbulência no Ambiente 28

Figura 3: Níveis de Incerteza $\quad 30$

Figura 4: Previsões de Consumo de Energia na Holanda 34

Figura 5: O Triângulo Grego 37

Figura 6: Futuros Possíveis, Realizáveis e Desejados 40

Figura 7: Esquema de Construção de Cenários Exploratórios 42

$\begin{array}{ll}\text { Figura 8: } & \begin{array}{l}\text { Equilíbrio entre Previsibilidade e Incerteza no Ambiente de } \\ \text { Negócios }\end{array} 48\end{array}$

Figura 9: Método de Cenários da Escola Francesa 55

Figura 10: Diagrama de Motricidade x Dependência 56

Figura 11: Método de Cenários da Escola Anglo-saxônica 58

Figura 12: Matriz de Impacto x Incerteza 60

Figura 13: Matriz de Combinação de Incertezas 60

Figura 14: Estratégias Deliberadas e Emergentes 72

Figura 15: Evolução de Escolas de Pensamento Estratégico 87

Figura 16: Quatro Visões do Processo de Formulação de Estratégia 87

Figura 17: Perspectivas Genéricas de Estratégia 88

Figura 18: Matriz SWOT para a Formulação de Estratégias segundo
Weihrich

Figura 19: Matriz de Avaliação Estratégica 93

Figura 20: Tipos de Estratégia por Quadrante 94

Figura 21: Modelo das Cinco Forças Competitivas 96

Figura 22: Tipos de Análise de Stakeholders 99

$\begin{array}{cc}\text { Figura 23: Processo de Análise de Stakeholders centrado na } & 100 \\ \text { Organização } & \end{array}$

Figura 24: Mapa dos Stakeholders da Atividade de Transmissão da Chesf 101

Figura 25: Diagnóstico de Stakeholders Organizacionais 104

Figura 26: Modelo de Aprendizagem Experimental 114 
Figura 27: Integração das Três Escolas de Estratégia

Figura 28: Aprendizagem Organizacional segundo Argyris e Schön

Figura 29: Principais Usos do Planejamento sob Cenários

Figura 30: Planejamento Estratégico com base em Cenários: a abordagem integrada

Figura 31: Visão geral do Processo TAIDA ${ }^{\text {TM }} 128$

Figura 32: Administração de Questões Estratégicas

Figura 33: Essência do Modelo Integrador - dos Cenários às Estratégias Múltiplas

Figura 34: Cenários Relativos à Adoção de um Novo Padrão de Sistema Operacional nos EUA no Início da Década de 90

Figura 35: Portfolio de Experimentos da Microsoft com Base em Cenários

Figura 36: Versão Inicial do Modelo Integrador Detalhado - das Estratégias às Estratégias Múltiplas

Figura 37: Construção da Estratégia em face dos Cenários

Figura 38: Análise de Sinais para Gerenciamento de Estratégias Múltiplas

Figura 39: Delineamento da Pesquisa - Macroetapas

Figura 40: Tipos Básicos de Projetos para Estudos de Caso

Figura 41: Detalhamento das Etapas da Pesquisa

Figura 42: Composição do Sistema Eletrobras

Figura 43: Metodologia Macroplan de Construção de Cenários

Figura 44: Diagrama Motricidade-Dependência do Ambiente de Interesse do Sistema Eletrobras

Figura 45: Cenários do Ambiente de Interesse do Sistema Eletrobras 2010-2030

Figura 46: Modelo das Cinco Forças Competitivas como uma Focalização dos Cenários

Figura 47: Análise da Futura Estrutura da Indústria do Segmento Geração de Energia Elétrica Associada aos Cenários 1 e 2

Figura 48: Análise da Futura Estrutura da Indústria do Segmento Geração de Energia Elétrica Associada aos Cenários 3 e 4

Figura 49: Stakeholders Segundo o Grau de Cooperação e de Ameaça por Cenário

Figura 50: Análise SWOT do Sistema Eletrobras por Cenário

Figura 51: Insights de Estratégias para Capturar Oportunidades

Figura 52: Posicionamento Estratégico do Sistema Eletrobras

Figura 53: Teste de Robustez do Plano Estratégico

Figura 54: Versão Final do Modelo Integrador Detalhado - dos Cenários às Estratégias Múltiplas 


\section{Lista de Quadros}

Quadro I: $\quad$ O Portfolio de Experimentos da Microsoft 74

$\begin{array}{lll}\text { Quadro II: } & \text { Posturas de Planejamento } & 108\end{array}$

Quadro II: $\quad$ Comparações entre o Planejamento Tradicional e o Planejamento sob Cenários $\quad 125$

Quadro V: $\quad$ Níveis de Incerteza, Postura Estratégica e Portfolio de Ações

Quadro V: $\quad$ Mudanças Ambientais, Implicações para o Planejamento e Estratégia Recomendada para a Empresa 132

Quadro VI: $\quad$ Postura Estratégica e Escolha em face dos Cenários 159

Quadro VII: Táticas do Estudo de Caso para Quatro Testes de Projeto 181

Quadro VIII: Dimensões, Variáveis e Atores considerados nos Cenários 201

Quadro IX: Condicionantes do Futuro do Ambiente de Interesse do Sistema Eletrobras

Quadro X: $\quad$ Elementos Essenciais dos Cenários: Situação em 2030206

Quadro XI: $\quad$ Das Forças Competitivas aos Insights de Estratégia Geração de Energia Elétrica no Cenário 2 - Expansão Incentivada - Exemplo

Quadro XII: Natureza da Relação e Pontos Críticos para Stakeholders Selecionados no Cenário 3: Concorrência na Escassez Exemplo

Quadro XIII: Natureza, Pontos Críticos e Insights de Estratégias para o Cenário 3: Concorrência na Escassez - Exemplo

Quadro XIV: Dimensões e Subdimensões para Consolidação dos Insights de Estratégia por Cenário

Quadro XV: $\quad$ Modelo para Seleção do Portfolio de Experimentos

Quadro XVI: Avaliação do Processo de Planejamento Corporativo do Sistema Eletrobras

Quadro XVII: Objetivos Estratégicos Finalísticos e Estratégias Associadas

Quadro XVIII: Objetivos Estratégicos de Gestão e Competências e Estratégias Associadas 


\section{Lista de Tabelas}

Tabela I: Composição do Grupo Participante da Formulação do Plano Estratégico

Tabela II: Pontos Fortes e Fracos relativos a Avaliação do Módulo I 257

Tabela III: Pontos Fortes e Fracos relativos a Avaliação do Módulo II 258

Tabela IV: Pontos Fortes e Fracos relativos a Avaliação do Módulo III 259

Tabela V: $\quad$ Pontos Fortes e Fracos relativos a Avaliação do Módulo IV 259

Tabela VI: Avaliação da Metodologia Empregada no Processo de Planejamento do Sistema Eletrobras 